\title{
Co-Injection of patient-derived fibroblasts enhances tumor growth, stromal invasion, and epithelial-to- mesenchymal transition
}

Ines Lohse ${ }^{1}$, Christine Crossman ${ }^{1}$, Keira Pereira ${ }^{1,2}$, Andrew Clayton Haller ${ }^{1,3}$, Stephen Chung ${ }^{1}$, Melania Pintilie ${ }^{1}$, Amy Jackson-Fisher ${ }^{4}$, Todd van Arsdale ${ }^{4}$, Laurie E. Ailles ${ }^{1,2}$, Catherine O'Brien ${ }^{1,3,5}$, Bradly G. Wouters ${ }^{1,2,6}$ and David W. Hedley ${ }^{1,2,7,8^{*}}$

*Correspondence: david.hedley@uhn.on.ca

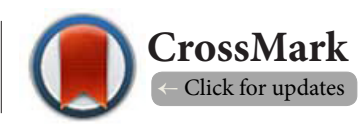

'Ontario Cancer Institute and Campbell Family Cancer Research Institute, Princess Margaret Cancer Center, University Health Network, 610 University Ave., Toronto, ON M5G2M9, Canada.

${ }^{2}$ Departments of Medical Biophysics University of Toronto, 610 University Ave., Toronto, ON M5G2M9, Canada.

${ }^{3}$ Department of Laboratory Medicine and Pathobiology, University of Toronto, Toronto, Ontario, Canada.

${ }^{4}$ Pfizer, Oncology Research Unit, 10724 Science Center Drive, 92121San Diego, CA, USA.

${ }^{5}$ Department of Surgery, Toronto General Hospital, Toronto, Ontario, Canada.

${ }^{6}$ Department of Radiation Oncology University Health Network, 610 University Ave, Toronto, ON M5G 1L7, Canada.

${ }^{7}$ Department of Medicine, University of Toronto, 610 University Ave., Toronto, ON M5G2M9, Canada.

${ }^{8}$ Department of Medical Oncology and Hematology, Princess Margaret Cancer Center, 610 University Ave., Toronto, ON M5G2M9,

Canada.

\begin{abstract}
Tumor-stroma interactions are regulated through a network of autocrine and paracrine signaling pathways that are crucial for many aspects of tumor development. Activation and signaling through the HGF/Met pathway have been shown to increase cell motility and reduce sensitivity to chemotherapy. We used a patient-derived colon-cancer epithelial stem cell line and patient-derived normal fibroblasts (NFs) or cancer-associated fibroblasts (CAFs), chosen for high HGF expression, to investigate the contribution of stromal signaling through the MET pathway in tumor growth and sensitivity to the Met inhibitor crizotinib. Co-injection of the colon cancer cell line with NFs or CAFs resulted in significantly increased tumor take. Additionally, tumors derived from the co-injections showed increased growth rates, an invasion of host stroma, increased expression of vimentin, and reduced sensitivity to crizotinib. These data demonstrate that stromal signaling through HGF/Met from adjacent fibroblasts leads to a more aggressive tumor phenotype, and can cause resistance to treatments targeting this pathway.
\end{abstract}

Keywords: EMT, crizotinib, colorectal cancer, cancer-associated fibroblasts

\section{Introduction}

Tumor growth, progression, and metastasis are supported by the tumor-associated stroma [1-7]. Tumor-associated stroma mainly consists of tumor-associated fibroblasts, extracellular matrix (ECM), basement membrane and vasculature that are recruited from host tissue. While stromal cells may initially display tumor suppressing characteristics [8], these cells undergo significant changes during tumor progression that promote growth and metastasis through a crosstalk between cancer cells and tumor-associated stroma cells $[\mathbf{1 , 3 , 4 , 7}]$. The most relevant change is the transdifferentiation of normal fibroblasts (NFs) to cancer-associated fibroblasts (CAFs) which is driven by cancerderived cytokines [9]. CAFs are characterized by the expression of smooth-muscle actin (SMA) and stroma-derived factors such as the hepatocyte growth factor (HGF) $[\mathbf{9}, \mathbf{1 0}]$. HGF, also known as scattering factor, is a ligand for Met tyrosine kinase. The activation of Met through HGF binding alters tumor cell motility and has been shown to induce chemotherapy resist- 
ance by protecting tumor cells from apoptosis, and to promote angiogenesis in the hypoxic microenvironment [10-12]. In addition to its action on cancer cells via paracrine mechanisms, HGF also acts on CAFs through an autocrine mechanism that benefits tumor progression [12].

We have recently shown that tumor hypoxia correlates with rapid growth and high metastatic potential in patient-derived pancreatic xenograft models [13]. Additional studies suggest that high levels of hypoxia in the stromal fraction play an important role in facilitating tumor metastasis [14]. This may be due to a dysregulated activation of the HGF/Met pathway in areas of extreme hypoxia within the tumor.

Based on the importance of the HGF/Met pathway, some Met inhibitors have been developed over the last few years. One of these agents, crizotinib, is fairly selective towards c-Met and ALK, and has clinical activity against non-small cell lung cancers that express mutant ALK. Crizotinib has been shown to inhibit the growth and survival of cell lines dependent upon c-Met or ALK enzymatic activity [15-17].

In the present study, we investigated the consequences of the co-injection of patient-derived NFs and CAFs producing HGF on the growth and microenvironment of a patient-derived colon cancer stem cell line and its effect on sensitivity to crizotinib.

\section{Materials and methods Cell Culture}

\section{Patient-derived colon cancer stem cell line}

POP92 cells were derived from a primary xenograft, established in the laboratory of Dr O'Brien from a surgically-resected liver metastasis from a 45-year-old patient with metastatic poorly differentiated colon adenocarcinoma, and maintained in the flanks of NOD/SCID mice. Genomic analysis of the patient's tumor identified mutations in APC, TP53, and BRAF that were retained in the xenografts, along with CK20 positivity. Second generation xenografts were excised, disrupted and the patientderived stem cell line POP92 was established and cultured as described previously [18]. Briefly, the spheroids were grown in the stem cell medium on ultra-low attachment surface dishes (Corning). For passaging, spheres were disrupted using 1x Trypsin.

\section{Patient-derived fibroblasts}

All human tissue procurement for these experiments was obtained in compliance with the institutional guidelines provided by the Institutional Review Board of the University Health Network. Tumor and adjacent normal tissue specimens were obtained from individuals undergoing surgical resection at Toronto General Hospital. Primary cancer-associated fibroblasts and normal adjacent fibroblasts were isolated from these tissues as follows. Tumor and normal tissue were chopped up and dissociated for $2 \mathrm{hr}$ at $37^{\circ} \mathrm{C}$ in Medium 199 containing collagenase/hyaluronidase (Stem Cell Technologies, Vancuver, BC, CA), DNase 1 (Worthington Biochemical Corp., Lakewood, NY, USA) and ROCK inhibitor (Cedarlane, Burlington, ON, CA). The slurry was triturated approximately every 20 minutes until no large chunks were observed and filtered through a $70 \mu \mathrm{m}$ strainer (BD, Mississauga, ON, CA). The single-cell suspension was then centrifuged at $1500 \mathrm{rpm}$ for 5 minutes. The cell pellet was resuspended in ACK lysis buffer (Gibco Life Technologies, Burlington, $\mathrm{ON}, \mathrm{CA}$ ) and incubated on ice for 5 minutes to remove red blood cells. The cells were washed with Hank's buffered salt solution (Wisent Bioproducts, ST-Bruno, QC, CA) containing $2 \%$ fetal bovine serum and pelleted by centrifugation. The cells were then resuspended in Iscove's Modified Dulbecco's Medium (Wisent Bioproducts, ST-Bruno, QC, CA) containing 10\% FBS (Gibco Life Technologies, Burlington, ON, CA) and 1\% penicillin/streptomycin (Gibco Life Technologies, Burlington, ON, CA) (IMDM10). An aliquot of cells was plated in a 6-well tissue culture treated plate. IMDM10 is selective for the outgrowth of fibroblasts from this tissue. Tumor and normal epithelial cells do not survive after the first passage. We verified that NF and CAF cultures were cytokeratin negative and vimentin positive using immunohistochemistry, and negative for CD45 and CD31 using flow cytometry.

The normal fibroblast (90967 NF) and tumor-associated fibroblast (61137 CAF) cell lines were subsequently cultured in a monolayer in IMDM media supplemented with $1 \%$ penicillin streptomycin and 10\% FBS at 3\% O2. Cells were cultivated for a maximum of 10 passages.

\section{Drug treatments}

Crizotinib was provided by Pfizer (New York, NY, USA), and crizotinib solution was prepared as described earlier [15]. Animals were treated with $50 \mathrm{mg} / \mathrm{kg}$ crizotinib daily by oral gavage for 14 days. For in vitro experiments, cells were treated withvehicle or $10 \mathrm{nM}, 25 \mathrm{nM}, 50 \mathrm{nM}$ or $100 \mathrm{nM}$ crizotinib for $24 \mathrm{~h}$ prior to protein extraction.

\section{In vitro evaluation of crizotinib target specificity}

Cells were cultured in a transwell assay setup to allow signaling between POP92 tumor cells and 90967 NF cells while keeping both cell lines separate for individual harvest and Western Blot analysis. $5 \times 10^{5} \mathrm{NF}$ cells were seeded into 6-well plates and incubated for $20 \mathrm{~h}$ prior to seeding of POP92 cells into the cell culture insert (8.0 $\mathrm{mm}, \mathrm{BD}$ Falcon, Franklin Lakes, NJ, USA). $4 \mathrm{~h}$ after POP92 seeding, vehicle or $10 \mathrm{nM}, 25 \mathrm{nM}, 50 \mathrm{nM}$ or $100 \mathrm{nM}$ crizotinib were added to both compartments of the Transwell assay and cells incubated for $24 \mathrm{~h}$. Cells were harvested using $1 \mathrm{x}$ Trypsin and cell pellets incubated in the cell lysis buffer for $1 \mathrm{~h}$ onice. $10 \mu \mathrm{g}$ of total protein was separated on an $8 \%$ SDS gel and transferred to PVDF membrane (GE Healthcare, BaiedUrfe, QC, CA). The membrane was first probed using the anti-pMet (Tyr1n7349) antibody (Cell signaling technologies, Danvers, MA, USA, clone 130H2, 1:1000), stripped and reprobed using the anti-Met antibody (Cell signaling technologies, Danvers, MA, USA, clone 25H2, 1:1000). $\beta$-actin (Abcam, Toronto, ON, CA, clone AC-15, 1:1000) was used as loading control. 


\section{Xenograft assay}

To investigate the effect of NF and CAF cells on tumor growth 4-5 week old SCID mice were injected with $4 \times 10^{5}$ POP92 cells in the right flank and a suspension of $2 \times 10^{5} \mathrm{NF}$ or CAF cells and $2 \times 10^{5}$ POP92 cells on the left flank. Animals were sacrificed 33days after injection when the tumors reached $\sim 1.5 \mathrm{~cm}$ in diameter.

To investigate whether the co-injection of NF affects the response to the Met-inhibitor crizotinib, SCID mice were injected with either $1 \times 10^{6}$ POP92 cells or a suspension of $5 \times 10^{5}$ $\mathrm{NF}$ and $5 \times 10^{5}$ POP92 cells into the right flank. Animals were randomized, and treatment started when tumors reached approximately $200 \mathrm{~mm}^{3}$.

Tumors grown subcutaneously were measured three times a week using callipers, and their volume calculated according to the formula width $2 \times$ length $\times 0.5$. Tumor weight was determined at the endpoint.

\section{Immunohistochemistry}

To determine the level of tumor hypoxia, mice were injected intraperitoneally (ip) with the 2-nitroimidazole EF5, $30 \mathrm{mg} / \mathrm{kg}$, three $h$ prior to sacrifice $[19,20]$. Tumors were excised, fixed, and paraffin embedded, and tissue sections were dried in $60^{\circ} \mathrm{C}$ oven overnight before staining. Slides were dewaxed in xylene and rehydrated through graded alcohol to water.

For EF5 staining, endogenous peroxidase was blocked in 3\% hydrogen peroxide for $10 \mathrm{~min}$. Microwave antigen retrieval was carried out under pressure at $120^{\circ} \mathrm{C}$ for $10 \mathrm{~min}$ in $10 \mathrm{mM}$ citrate buffer, pH 6.0 using a T/T Mega microwave oven. Slides were cooled to room temperature before removal. Endogenous biotin was blocked in Vector's biotin blocking kit, and slides incubated in biotinylated EF5 antibody (1:250, obtained from Dr. Cameron Koch, University of Pennsylvania) at overnight. After incubation in HRP labeling reagent (Signet Pathology System) for 30min, immunoreactivities were revealed by incubation in Nova Red substrate (Vector Labs, Burlington, ON, Canada) for $5 \mathrm{~min}$ and counterstained in Mayer's haematoxylin.

For vimentin (DAKO, Glostrup, Denmark, clone vim 3B4, 1:1000), a-Smooth Muscle Actin (DAKO, Glostrup, Denmark, clone 1A4, 1:400), CD31 (Santa Cruz, Dallas, TX, USA, clone sc-1506,1:1000) and phospho Met (Tyr 1234/1235) (pMet) (Cell signaling technologies, Danvers, MA, USA, clone 3077, 1:50), E-Cadherin (Vector Labs, Burlington, ON, Canada, VP-E601, 1:50), N-Cadherin (BD, Franklin Lakes, NJ, USA, 610921 Clone $32,1: 50)$ stainings, the immunohistochemistry was performed according to the manufacture's guidelines in the BenchMark XTan automated slide strainer (Ventana Medical System, Tucson, AZ, USA) using VentanaUltraview Universal DAB Detection Kit (Ventana Medical System, Tucson, AZ, USA). The slides were counterstained with Ventana Hematoxylin II and Bluing reagent (Ventana Medical System, Tucson, AZ, USA). Sections were scanned at 20x resolution using an Aperio Scanscope XT scanner (Aperio Technologies, Vista, CA, USA). Images were analyzed using the Aperio Imagescope software (Vs. 11.1.2.752, Aperio Technologies, Vista, CA, USA). Necrotic areas were excluded from the analysis, and the V9 algorithm was then used as a base and adapted using a control sample in order to accommodate differences between cytoplasmic, nuclear and membrane staining.

\section{HGF elisa}

Tumor pieces of POP92, POP92-NF, and POP92-CAF tumors were homogenized for $2 \mathrm{~min}$, followed by centrifugation at $800 \mathrm{~g}$ to remove intact cells and debris. HGF expression in the tumor lysates was evaluated using the Quantikine ELISA Human HGF Immunoassay Kit (R\&D Systems, Minneapolis, MN, USA) according to the manufactures' guidelines.

\section{Statistical analysis}

To test the differences between the growth rates in the different groups, mixed effect modeling was employed on the data points for which the tumor was measurable. The tumor volumes were transformed (cubic root) to normalize the residuals. For the analysis of growth delay the time to the point on which the tumor reached $1000 \mathrm{~mm}^{3}$ was recorded. The tumors that did not reach that size were considered censored. These data were analyzed by employing time to event techniques (Kaplan-Meier curves). The curves were compared using the likelihood ratio test (LRT). The markers were tested for the differences between groups using nonparametric tests - Kruskal-Wallis for multiple group comparison and Mann-Whitney for two group comparisons.

\section{Results}

\section{Co-Injection of POP92 and NFs or CAFs results in rapid tumor occurrence and increased take rate}

A balanced co-injection of POP92 and NF cells results in rapid tumor initiation $(\mathrm{p}=0.026)$ and increased tumor weight $(\mathrm{p}=0.056)$ when compared to injection of POP92 alone. A similar increase was observed in the co-injection of POP92 and CAF cells (tumor initiation ( $p=0.0063)$, tumor weight $(p=0.016)$ ) (Figures 1A and 1B). Furthermore, co-injection of POP92 and NF/CAF cells increased tumor take rate (Figures $1 \mathrm{~A}$ and $1 \mathrm{C}$ ). While injection of $4 \times 10^{5}$ POP92 alone gave rise to tumors in $50 \%$ of the injected mice, the co-injection with either $2 \times 10^{5} \mathrm{NF}$ or CAF and $2 \times 10^{5} \mathrm{POP} 92$ cells increase tumor take to $100 \%$ (Figure 1C).

Injection of either NF or CAF cells alone did not give rise to any tumors (Figures 1A-1C). While co-injection of POP92 with CAF cells increased tumor growth rate $(p=0.0032$; Figure 1A), co-injection of NF did not. However, the proportion of actively proliferating cells in the tumor as assessed by the Ki67 index was not increased by co-injection with either NF or CAF cells $(\mathrm{p}=0.11$; Supplementary figure $\mathrm{S} 1)$, suggesting that coinjection of NF or CAFs reduced tumor latency but not growth rate.

Although the co-injection of human fibroblasts had a large impact on initial tumor initiation and take rate, no stromal cells positive for human vimentin were found in the stromal compartment of endpoint tumors (Figures 2B and 2F). Consistent with this observation, no increase in human HGF was observed in tumors derived from the co-injection of POP92 and NF or 


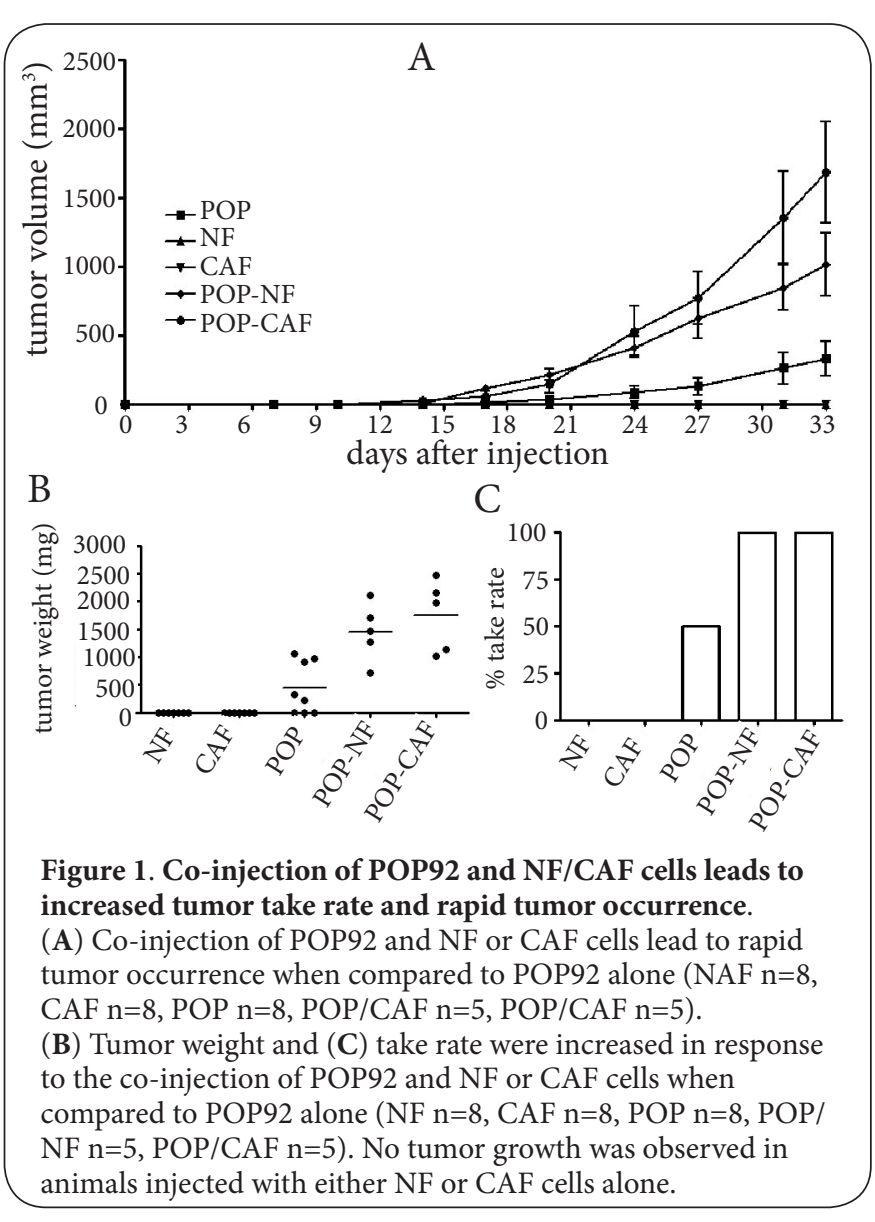

\section{CAF cells (Supplementary figure S1).}

\section{The presence of human fibroblasts increases the expression of tumor vimentin and leads to enhanced stromal invasion}

While no expression of human vimentin, indicating the presence of human fibroblasts, was observed in the stromal areas of tumors derived from the co-injection of POP92 and NF or CAF cells, vimentin expression in these tumors was significantly increased in the tumor compartment $(\mathrm{p}=0.0069)$ (Figures 2B and 2F). Consistent with the increased expression of vimentin, tumors derived from the co-injection of POP92 with NF and CAF cells results in reduced expression of E-Cadherin (Figures $\mathbf{2 C}$ and $\mathbf{2 G}$ ) and increased expression of N-Cadherin (Figure 2D+H) when compared to tumors derived from the injection of POP92 cells alone. Furthermore, co-injection of POP92 with NF cells led to a significant increase in SMA positive cells in the stromal compartment when compared to POP92 alone $(p=0.0087$; Figures $2 \mathrm{~A}$ and $2 \mathrm{E}$ ) indicating increased recruitment of mouse fibroblasts into the tumor.

Thus, the presence of stromal cells during tumor initiation had a lasting consequential effect on the microenvironment of the tumors after establishment. The extent of hypoxia assessed by EF5 staining was not significantly altered in tumors derived

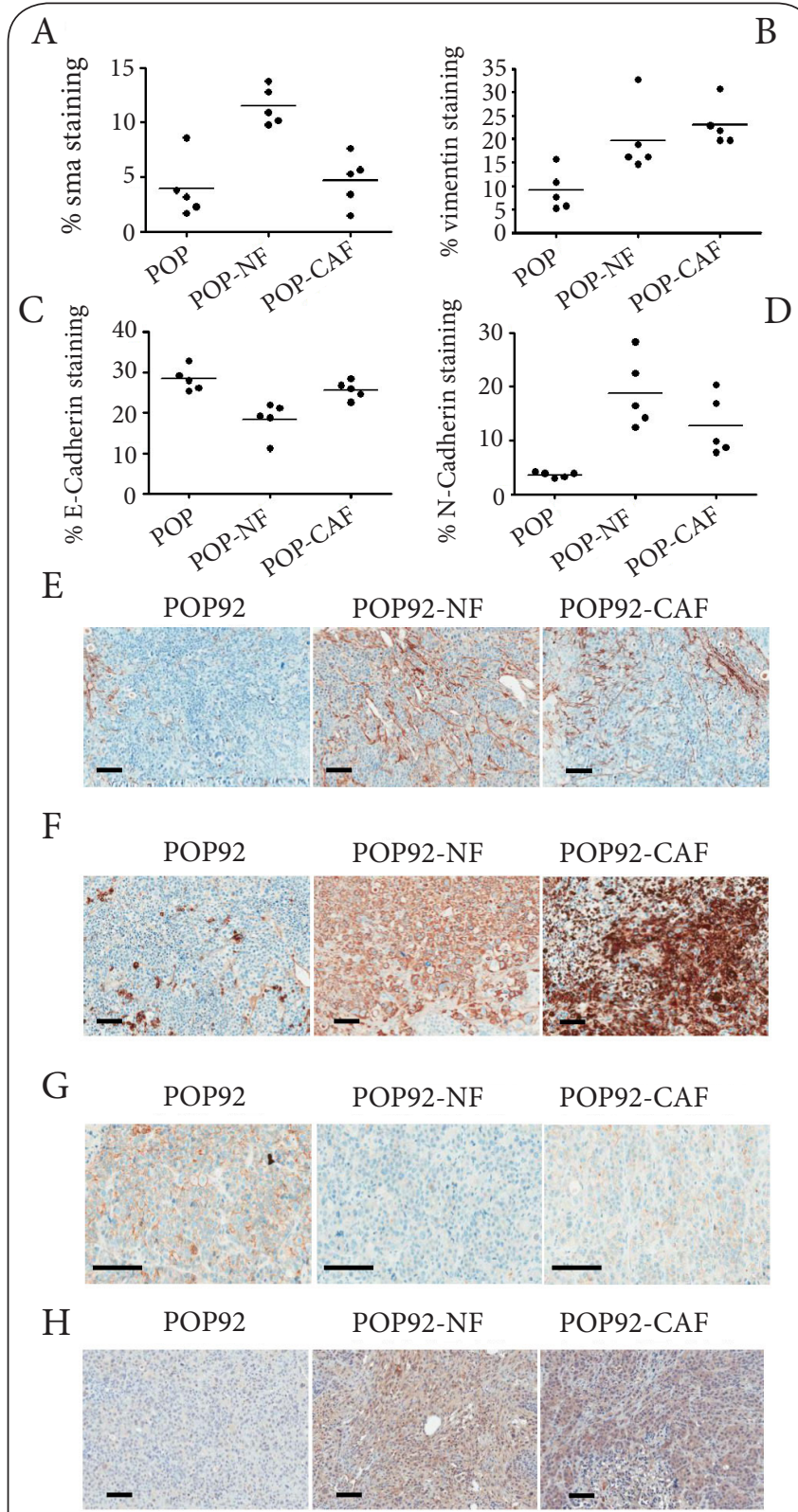

Figure 2: Co-injection of POP92 and NF/CAF cells results in increased Vimentin staining and stromal invasion.

$(\mathbf{A}+\mathbf{E})$ Smooth muscle actin, $(\mathbf{B}+\mathbf{F})$ Vimentin, $(\mathbf{C}+\mathbf{G})$

E-Cadherin and $(\mathbf{D}+\mathbf{H}) \mathrm{N}$-Cadherin expression were evaluated using immunohistochemistry (POP $n=5, P O P / N F n=5, P O P /$

CAF $n=5)$. Bars represent $100 \mu \mathrm{m}$.

from the co-injection when compared to tumors derived from POP92 injections ( $\mathrm{POP}+\mathrm{NF}$ vs $\mathrm{POP}, \mathrm{p}=0.25$; $\mathrm{POP}+\mathrm{CAF}$ vs $\mathrm{POP}$, $\mathrm{p}=0.56$; Supplementary figure $\mathrm{S} 1$ ).

\section{Co-Injection of NFs reduces sensitivity to the met- inhibitor crizotinib}

Crizotinib target specificity in vitro was evaluated using POP92 cells in monoculture and a co-culture of POP92 and NFs. Cells were treated with vehicle or $10,25,50$ or $100 \mathrm{nM}$ crizotinib 
for $24 \mathrm{~h}$. Total protein was extracted, and pMet and total Met expression were analyzed by Western Blot. When compared to POP92 monoculture, POP92 cells that were co-cultured with NFs show increased expression of both phosphorylated and total Met (Figure 3A). Crizotinib treatment leads to a dose-dependent reduction in Met phosphorylation in both POP92 monoculture and co-culture with NFs (Figure 3A). While a significant reduction of Met phosphorylation in the monoculture was observed in response to $25 \mathrm{nM}$ crizotinib, treatment with $50 \mathrm{nM}$ crizotinib was necessary to achieve a similar reduction in co-cultured POP92 cells (Figure 3A).

In order to examine the effect of crizotinib in vivo, animals were injected with either POP-92 alone or a suspension of POP92 and NF and treated with $50 \mathrm{mg} / \mathrm{kg}$ Crizotinib for 14 days once the tumor volume reached $\sim 150 \mathrm{~mm}^{3}$. Similar to the results shown in Figure 1A, co-injection of POP92 and NF cells resulted in faster tumor occurrence $(p=0.041)$ and growth rate $(p=<0.0001$; Figure $3 \mathbf{B})$. Treatment with crizotinib had no impact on tumor growth in animals co-injected with POP92 and NF cells ( $p=0.47$; Figure 3 B). Treatment of animals injected with POP92 alone however resulted in a modest non-significant smaller tumor growth rate $(\mathrm{p}=0.083$; Figure 3B). Consistent with the in vitro treatment, treatment of tumors derived from POP92 alone resulted in a significant reduction of Met phosphorylation when compared to vehicle control $(p=0.03)$. However, tumors derived from the co-injection of POP92 and NF cells displayed no reduction in

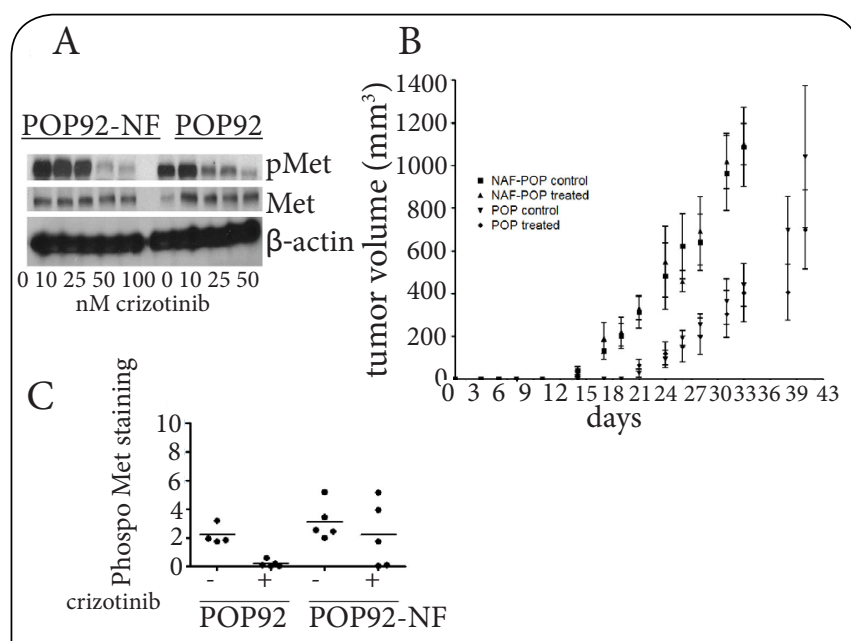

Figure 3: Co-injection of POP92 and NF cells reduced crizotinib efficiency.

(A) Western Blot analysis of POP92 cells cultured in the presence or absence of NFs treatment. (B) While treatment with crizotinib leads to a mild growth delay in mice injected with POP92 alone, no growth delay was observed in tumors derived from co-injection of POP92 and NF cells (POP/NF treated $n=5, P O P / N F$ control $n=5$, POP treated $n=5$, POP control $n=5)$. (C) Met phosphorylation in response to crizotinib treatment was evaluated using immunohistochemistry in POP92 tumors (POP/NF treated $n=5$, POP/NF control $n=5$, POP treated $n=5$, POP control $\mathrm{n}=5$ ).
pMet in response to crizotinib treatment (Figure 3C).

\section{Discussion}

CAFs, similar to immune cells, are a subpopulation of cells within the tumor microenvironment whose role in tumor progression is multifaceted [9-11]. While CAFs have been shown to inhibit the early stages of tumor progression [8], these cells have also been shown to promote tumor growth, angiogenesis and metastasis once activated through signals secreted by the tumor cells $[1-7,9,10]$.

The results presented here demonstrate that co-injection of human fibroblasts significantly increases tumor initiation and take rate. Interestingly, tumors derived from the coinjection displayed higher stromal content and increased vimentin expression in the tumor compartment, suggesting that the presence of fibroblasts during early tumor growth promotes an EMT phenotype in this model, even though no human fibroblasts were present in endpoint tumors. The loss of human fibroblasts during most of tumor growth may also explain why no impact on growth rate was observed in response to coinjection of human NFs and CAFs.

Our results show that crizotinib substantially reduced Met phosphorylation in POP92 tumors, even though no growth delay was observed. No reduction in pMet was observed in tumors derived from the coinjection of POP92 and NF cells. Although no human fibroblasts were found in these tumors, the coinjection of NF and CAF cells had long-lasting effects on the tumor phenotype that can be attributed to HGF secretion by the tumor-associated stroma at the time of tumor initiation. Our results shed some light on adisconnect between preclinical testing and clinical trials of crizotinib. As seen in tumors derived from the POP92 injection, mouse CAFs can support tumor growth and Met phosphorylation. Nevertheless, mouse HGF is not as potent as the human counterpart and exposure to human NFs reduces crizotinib sensitivity.

Improved preclinical models will be essential to predict reliably the efficacy of novel treatment modalities that disrupt tumor-stroma interactions.

\section{Additional files}

\section{Supplementary figure $\mathbf{S} 1$}

\section{Competing interests}

The authors declare that they have no competing interests.

\section{Author contributions}

IL, AJ-F, TvA, BGW, DWH: experimental design

IL, CC, KP, ACH, SC: experimental procedures

MP: statistical analysis

IL, AJ-F, TvA, LEA, CO, BGW, DWH: manuscript preparation.

\section{Acknowledgement}

Crizotinib was provided by Pfizer.

\section{Publication history}

Editor: Paul J. Higgins, Albany Medical College, USA.

Received: 03 May 2015 Revised: 29 July 2015

Accepted: 01 August 2015 Published: 06 August 2015 
Hedley et al. Journal of Cancer Therapeutics \& Research 2015,

\section{References}

1. Bhowmick NA, Neilson EG and Moses HL. Stromal fibroblasts in cancer initiation and progression. Nature. 2004; 432:332-7. | Article | PubMed Abstract | PubMed FullText

2. Chu GC, Kimmelman AC, Hezel AF and DePinho RA. Stromal biology of pancreatic cancer. J Cell Biochem. 2007; 101:887-907. | Article | PubMed

3. De Wever $O$ and Mareel $M$. Role of tissue stroma in cancer cell invasion. J Pathol. 2003; 200:429-47. | Article | PubMed

4. De Wever O, Demetter P, Mareel M and Bracke M. Stromal myofibroblasts are drivers of invasive cancer growth. Int J Cancer. 2008; 123:2229-38. | Article | PubMed

5. Kuperwasser C, Chavarria T, Wu M, Magrane G, Gray JW, Carey L, Richardson $A$ and Weinberg RA. Reconstruction of functionally normal and malignant human breast tissues in mice. Proc Natl Acad Sci U S A. 2004; 101:4966-71. | Article | PubMed Abstract | PubMed FullText

6. Olumi AF, Grossfeld GD, Hayward SW, Carroll PR, TIsty TD and Cunha GR. Carcinoma-associated fibroblasts direct tumor progression of initiated human prostatic epithelium. Cancer Res. 1999; 59:5002-11. | Article | PubMed

7. Ostman A and Augsten M. Cancer-associated fibroblasts and tumor growth--bystanders turning into key players. Curr Opin Genet Dev. 2009; 19:67-73. | Article | PubMed

8. Rhim AD, Oberstein PE, Thomas DH, Mirek ET, Palermo CF, Sastra SA, Dekleva EN, Saunders T, Becerra CP, Tattersall IW, Westphalen CB, Kitajewski J, Fernandez-Barrena MG, Fernandez-Zapico ME, lacobuzioDonahue C, Olive KP and Stanger BZ. Stromal elements act to restrain, rather than support, pancreatic ductal adenocarcinoma. Cancer Cell. 2014; 25:735-47. | Article | PubMed Abstract | PubMed FullText

9. Peng $Y$, Li Z, Yang P, Newton IP, Ren H, Zhang L and Wu H. Direct contacts with colon cancer cells regulate the differentiation of bone marrow mesenchymal stem cells into tumor associated fibroblasts. Biochem Biophys Res Commun. 2014; 451:68-73. | Article | PubMed

10. De Veirman K, Rao L, De Bruyne E, Menu E, Van Valckenborgh E, Van Riet I, Frassanito MA, Di Marzo L, Vacca A and Vanderkerken K. Cancer associated fibroblasts and tumor growth: focus on multiple myeloma. Cancers (Basel). 2014; 6:1363-81. | Article | PubMed Abstract | PubMed FullText

11. Hwang $\mathrm{Cl}$, Choi J, Zhou Z, Flesken-Nikitin A, Tarakhovsky A and Nikitin AY. MET-dependent cancer invasion may be preprogrammed by early alterations of $p 53$-regulated feedforward loop and triggered by stromal cell-derived HGF. Cell Cycle. 2011; 10:3834-40. | Article | PubMed Abstract | PubMed FullText

12. Wu X, Chen X, Zhou Q, Li P, Yu B, Li J, Qu Y, Yan J, Yu Y, Yan M, Zhu Z, Liu B and Su L. Hepatocyte growth factor activates tumor stromal fibroblasts to promote tumorigenesis in gastric cancer. Cancer Lett. 2013; 335:12835. | Article | PubMed

13. Chang Q, Jurisica I, Do T and Hedley DW. Hypoxia predicts aggressive growth and spontaneous metastasis formation from orthotopically grown primary xenografts of human pancreatic cancer. Cancer Res. 2011; 71:3110-20. | Article | PubMed

14. Lohse I, Lourenco C, Ibrahimov E, Pintilie M, Tsao MS and Hedley DW. Assessment of hypoxia in the stroma of patient-derived pancreatic tumor xenografts. Cancers (Basel). 2014; 6:459-71. | Article | PubMed Abstract I PubMed FullText

15. Tanizaki J, Okamoto I, Okamoto K, Takezawa K, Kuwata K, Yamaguchi H and Nakagawa K. MET tyrosine kinase inhibitor crizotinib (PF-02341066) shows differential antitumor effects in non-small cell lung cancer according to MET alterations. J Thorac Oncol. 2011; 6:1624-31. | Article | PubMed

16. Ou SH. Crizotinib: a novel and first-in-class multitargeted tyrosine kinase inhibitor for the treatment of anaplastic lymphoma kinase rearranged non-small cell lung cancer and beyond. Drug Des Devel Ther. 2011; 5:471-85. | Article | PubMed Abstract | PubMed FullText
17. Heigener D.F and Reck M. Crizotinib. Recent Results Cancer Res. 2014; 201:197-205.

18. Kreso A and O'Brien CA. Colon cancer stem cells. Curr Protoc Stem Cell Biol. 2008; Chapter 3:Unit 3 1. | Article | PubMed

19. Koch CJ. Measurement of absolute oxygen levels in cells and tissues using oxygen sensors and 2-nitroimidazole EF5. Methods Enzymol. 2002; 352:3-31. | PubMed

20. Lord EM, Harwell L and Koch CJ. Detection of hypoxic cells by monoclonal antibody recognizing 2-nitroimidazole adducts. Cancer Res. 1993; 53:5721-6. | Article | PubMed

\section{Citation:}

Lohse I, Crossman C, Pereira K, Haller A.C, Chung S, Pintilie M, Jackson-Fisher A, Arsdale T.V, Ailles A.E, O'Brien C, Wouters B.G and Hedley D.W. Co-Injection of patient-derived fibroblasts enhances tumor growth, stromal invasion, and epithelial-to-mesenchymal transition. J Cancer Ther Res. 2015; 4:2.

http://dx.doi.org/10.7243/2049-7962-4-2 